\title{
The Outcome of Unintentional Pediatric Bupropion Ingestions: A NPDS Database Review
}

\author{
Michael C. Beuhler • Henry A. Spiller • Howell C. Sasser
}

Published online: 6 March 2010

(C) American College of Medical Toxicology 2010

\begin{abstract}
Unintentional bupropion pediatric exposures uncommonly report severe clinical effects such as seizures. We sought to determine the clinical effects and case outcomes for unintentional bupropion ingestions in children age $\leq 6$ years. The National Poison Data System was queried for unintentional, acute, single substance bupropion ingestions in children age $\leq 6$ years for the time period January 1, 2000 to February 27, 2007 for cases followed to a known outcome. If exposure amount was reported, a $\mathrm{mg} / \mathrm{kg}$ dose was determined; when weight was absent, it was interpolated from the available data set. An adverse neurological effect (ANE) was defined as seizure (single, multi/discrete, and status) or coma. For analysis, the outcomes of no effect and mild outcome were grouped, and the outcomes of moderate outcome, major outcome, and death were grouped. A subset of case notes were reviewed for accuracy. Seven thousand one hundred eighteen cases met the inclusion criteria, with 1,154 cases excluded because of multiple substances and coding
\end{abstract}

\section{Source of Funding None.}

Previous presentation as an abstract: Beuhler MC, Spiller HA. The outcome of unintentional pediatric bupropion ingestions: a TESS database review. Clin Tox 45(6):630, 2007

M. C. Beuhler $(\square)$

Carolinas Poison Center,

Carolinas Medical Center,

Charlotte, NC, USA

e-mail: michael.beuhler@carolinashealthcare.org

H. A. Spiller

Kentucky Regional Poison Center, Kosair Children's Hospital,

Louisville, KY, USA

\section{H. C. Sasser}

Dickson Institute for Health Studies Research Planning \& Evaluation, Carolinas Medical Center,

Charlotte, NC, USA errors, resulting in 5,964 cases. A total of 4,557 cases (76.4\%) were managed at or sent to a HCF. The most common clinical effects reported were nausea/vomiting (4.3\%), tachycardia (3.9\%), agitated/irritable (3.1\%), drowsiness/lethargy (2.4\%), and seizure (1.4\%). There were no deaths. Overall, there was a $3.3 \%$ rate of moderate/major outcomes. A $\mathrm{mg} / \mathrm{kg}$ dose was calculable in $76.1 \%$ of cases; the average amount for the no effect/minor cases and moderate/major effect was $13.8 \pm 18.8$ and $38.8 \pm 44.0 \mathrm{mg} / \mathrm{kg}$, respectively $(p<0.0001)$. Average time until development of seizures was $4.2 \pm 3.2 \mathrm{~h}$ with a maximum of $14 \mathrm{~h}$. Few children develop toxicity from unintentional reported bupropion ingestions, with about $1.5 \%$ of patients developing an ANE.

Keywords Bupropion · Pediatrics · NPDS · Poison centers · Unintentional

\section{Introduction}

Unintentional pediatric bupropion ingestions uncommonly report severe effects. However, seizures from relatively small ingestions have been reported, potentially resulting in significant morbidity. There is no clinical management consensus on unintentional bupropion cases, and many undergo gastric decontamination and/or are referred to a health care facility (HCF). We sought to determine the clinical effects, case outcomes, and decontamination therapies for unintentional bupropion ingestions in children age $\leq 6$ years.

\section{Methods}

The National Poison Data System (NPDS) was queried on March 2, 2007 for unintentional (general and unknown), 
acute bupropion exposures in children age $\leq 6$ that were followed up to a known outcome for the dates January 1, 2000 to February 27, 2007. Bupropion exposures were selected by using the American Association of Poison Control Centers (AAPCC) generic code "Other type of antidepressant" and all the known product codes for bupropion [1]. Cases not followed and cases where the outcome was coded as not responsible for the effect (these are outcomes of confirmed non-exposure and unrelated effect) were not included in the initial query. All cases with co-ingestants were excluded.

The AAPCC (http://www.aapcc.org/) maintains the national database of information logged by the U.S. Poison Control Centers (PCCs). Case records in this database are from selfreported calls; they reflect only information provided when the public or healthcare professionals report an actual or potential exposure to a substance (e.g., an ingestion, inhalation, topical exposure, etc.) or request information/ educational materials. Without further examination of case documentation, all reported exposures do not necessarily represent a poisoning or overdose. Although every effort was made to verify dosing in cases with major outcomes and seizures, not all of these cases demonstrated confirmation of the bupropion ingestion in the form of passed or vomited pills, positive drug screens, or blood bupropion levels. The AAPCC is not able to completely verify the accuracy of every report made to member centers. Additional exposures may go unreported to PCCs, and data referenced from the AAPCC should not be construed to represent the complete incidence of national exposures to any substance(s).

An adverse neurological effect (ANE) was defined as the case coding of any of the clinical effect such as seizures (single, multi/discrete, and status) or coma. These clinical effects were chosen based on the effects reported in the published case reports of bupropion toxicity in children. For analysis, the outcomes of no effect and mild outcome were grouped together, and the outcomes of moderate outcome, major outcome, and death were grouped together. Cases were included (regardless of management site) if their outcomes were no effect, mild, moderate, major, or death. A major outcome is defined as symptoms that are "life-threatening or resulted in significant residual disability or disfigurement." Moderate outcome is defined as symptoms that were "more pronounced, more prolonged or more of a systemic nature than minor symptoms" [2]. Management site was grouped into either HCF (already in or referred to HCF) or home/non-HCF. Cases were grouped based on two possible decontamination therapies coded as performed: activated charcoal (single-dose) or no gastric decontamination. For the no gastric decontamination group, cases were included if coded as no decontamination therapy or if the decontamination treatments were limited to dilute/ irrigate/wash, fresh air, and/or food/snack.
If an ingested amount was reported, a mg/ $\mathrm{kg}$ dose was determined. All products type (immediate release, sustained release, extended release, and unknown) were grouped together. This was because of the inability to reliably differentiate the type of products using the product code alone. It was documented in several of the reviewed case notes that the type of bupropion preparation was unknown, despite the coding of the case as a specific product type. As only a fraction of the total case notes could be reviewed, this prevented a detailed analysis based on the preparation type.

When weight was absent, it was interpolated from the available data set. The interpolation was performed by a bootstrap procedure using 100 samples per age stratum drawn without replacement, the size of each sample determined as a percentage of the valid recorded weights in that stratum $(60 \%$ was the standard for the majority of strata). The software SAS 9.1 (SAS Institute, Cary, NC, USA) was used for the analysis. This interpolation was acceptable because of the much lower overall variability in weight in this young pediatric population when compared to older children or adults, and this interpolation was based on a very similar set of patients, instead of relying on published tables that may not be representative of this population.

Dose estimations in NPDS are coded as estimate, exact, or maximum possible [2]. When the amount is specifically chosen to be maximum possible, this represents the upper (unlikely) extreme of what the ingested dose could have been. Commonly these cases reflected a history of ingestion of a number of tablets when some of the tablets are known to have been previously consumed (by the intended individual), or when some of the missing tablets are recovered but not countable (due to damage, etc.). In these situations, the actual dose is clearly less than the numerical amount reported. To adjust for this, when the amount was coded as maximum possible, the reported dose in these cases was multiplied by 0.5 . This multiplier was chosen because the true amount ingested is equal to the maximum possible amount times an unknown probability, which ranges from 0 to 1 . When the probability for an event is completely unknown, the use of 0.5 (the midpoint) for the probability estimate is better than using 1 ; this unbiased estimate minimizes the total population mean squared error. A consequence of this estimation will result in a more conservative approximation of dose-response effect.

Initial study design called for requesting the poison center case notes where seizures were coded and all the cases with outcome of major or death. However, after performing the initial data analysis, a minor number of cases with anomalies in the following variables were noted: age, weight, route, dose, and formulation. Because age and weights were used to build the interpolated age vs. weight 
data set, it was critical that there were no grossly incorrect weights with respect to age in the database. Weight anomaly was defined as when the coded weight was outside the 97th percentile expected on standard growth curves for the mid-point of the age bracket above or below the reported age [3]. Age was considered anomalous if the patient was reported to be less than 2 months of age because developmental limitations in fine motor grasp prevents an unintentional ingestion at that age. As the purpose of this investigation was to study cases of reported pediatric ingestion of bupropion, cases with routes coded as inhalation/nasal were considered suspect. Cases where the units of formulation were a liquid volume were unlikely as bupropion is only available as tablets. Finally, dose amounts that were believed to be excessive, such as a dose unit of pounds or grams or counts greater than 25 , were considered suspect. After getting a permission from the AAPCC, the case notes from these cases $(n=174)$ were requested from the individual poison centers.

All the returned case notes were reviewed by two authors (MCB and HAS). They identified the presence of several variables, including a subset of the clinical effects (seizures, coma, tachycardia, vomiting, agitated/irritable, hallucinations, tremor, ataxia, and drowsiness/lethargy), the ingested dose and type of dose estimate, the use of activated charcoal, patient age, patient weight, and time to seizure. Clinical effects and the presence of charcoal discussed in the case notes were added to the cases' coding for data analysis. Dosing amounts in the case notes replaced the originally coded amount. Seizure times were determined by case note interpretation and were recorded in hours. If the time of ingestion was not known, the seizure time was documented as unknown. If the seizure time was known to have occurred between two follow-up calls, the mid-point of that time span was used for when the seizure occurred, and the seizure time was considered an estimate.

The outcome groups were compared using two-tailed Fisher's exact and Mann-Whitney $U$ tests. Data was analyzed using SAS 9.1 (SAS Institute) and Microsoft Excel 2003 (Microsoft, Redmond, WA, USA). The study was deemed exempted by the host institution's institutional review board.

\section{Results}

Seven thousand one hundred eighteen cases met the inclusion criteria; there were no deaths. There were 1,143 cases excluded from this set because of multiple substances. Of the remaining cases, 174 cases contained a suspect variable, ANE or major outcome, and therefore, further review was pursued; all queried poison centers responded except one (center was closed). Of these cases, 168 (97\%) had some further case information provided by the original poison center; of these, there were 13 cases where the poison center only provided a summary of the case. Based on this secondary review, nine of the 168 cases were excluded for various reasons (wrong substance, polypharmacy, duplicate case, and wrong species). Regarding the six cases where further case information was not available for secondary review, there were two cases with major outcomes and four other cases. The two major outcomes were left in the database, the two improbable patient weights had a new weight calculated from the interpolated data and remained in the database, but the one improbable age and one nasal exposure were excluded from any further analysis. This left 5,964 cases for the final analysis (see Fig. 1 for the breakdown).

A total of 4,557 (76.4\%) cases were managed at or sent to a HCF. The most common clinical effects reported are found in Table 1. A mg/kg dose was calculable in $75.77 \%$ of cases; the average amount for the no effect/minor cases and moderate/major effect was $13.8 \pm 18.8$ and $38.8 \pm$ $44.0 \mathrm{mg} / \mathrm{kg}$, respectively; this difference was significant $(p<0.0001$; both parametric and non-parametric; see Table 2). The cases where an ANE was reported had an average dose of $73.6 \pm 54.3 \mathrm{mg} / \mathrm{kg}$. The outcomes and percentage of ANE with various reported $\mathrm{mg} / \mathrm{kg}$ exposure dosages are presented in Table 3. Activated charcoal was used in $50.1 \%$ of the included cases, with $44.5 \%$ of the cases receiving no gastric decontamination.

The average time from ingestion until the development of seizures for all the cases with times known with certainty

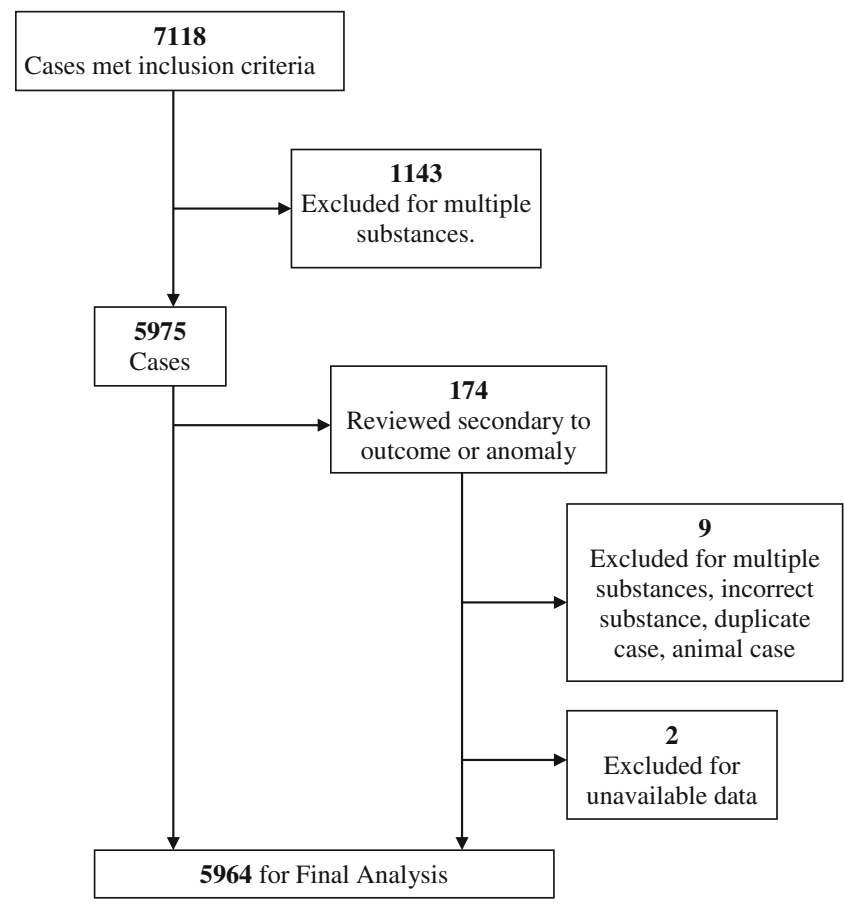

Fig. 1 Flow chart of study selection 
Table 1 Commonly reported clinical effects with bupropion exposure

\begin{tabular}{lc}
\hline Clinical effect & Rate of occurrence (\%) \\
\hline Any neuro symptom & 11.2 \\
Nausea/vomiting & 4.3 \\
Tachycardia & 3.9 \\
Agitated/irritable & 3.1 \\
Drowsiness/lethargy & 2.4 \\
ANE & 1.5 \\
Seizure & 1.4 \\
Tremor & 1.1 \\
Ataxia & 0.9 \\
Hallucinations & 0.6 \\
\hline
\end{tabular}

was $4.1 \pm 3.3 \mathrm{~h}$ with a range of 0.5 to $14 \mathrm{~h}(n=56)$. When the additional cases with estimated seizure times were included, the average time was $4.2 \pm 3.2 \mathrm{~h}$ with an identical range $(n=65$ total $)$.

\section{Discussion}

Bupropion is a dopamine and norepinephrine reuptake inhibitor that results in increased extracellular dopamine and norepinephrine concentrations [4]. It is used for treatment of depression, attention-deficit hyperactivity disorder, and smoking cessation. In overdose, it is known to cause altered mental status, seizures, arrhythmias, and death [5-7]. The spectrum of its clinical effects and appropriate recommendation for pediatric unintentional exposures is not well understood.

This is the largest collection of pediatric patients reported to be exposed by ingestion to bupropion, both in number of patients and years evaluated. This study attempts to answer the following question: "What should a Poison Center (or call center) recommend to a parent at home that calls with a child that was reported to unintentionally consume only bupropion?" Our data suggest that clinically significant effects are uncommon in this age group, that this is a dose-related event, and that not all of these patients require referral to an emergency department. The associa-
Table 3 Case outcome based on reported dose

\begin{tabular}{llll}
\hline $\begin{array}{l}\text { Reported } \\
\text { dose }\end{array}$ & Number & $\begin{array}{l}\text { Number with } \\
\text { moderate/major } \\
\text { outcomes (\%) }\end{array}$ & $\begin{array}{l}\text { Number of adverse } \\
\text { neurological events } \\
\text { (ANE) }\end{array}$ \\
\hline $\begin{array}{l}\text { Up to } \\
\quad 7 \mathrm{mg} / \mathrm{kg}\end{array}$ & 1,697 & 1 & 0 \\
$\begin{array}{l}\mathrm{Up} \text { to } \\
10 \mathrm{mg} / \mathrm{kg}\end{array}$ & 2,465 & 1.4 & $0.04^{\mathrm{a}}$ \\
$\begin{array}{l}\text { Up to } \\
12 \mathrm{mg} / \mathrm{kg}\end{array}$ & 3,077 & 1.5 & 0.1 \\
$\begin{array}{l}\text { Up to } \\
15 \mathrm{mg} / \mathrm{kg}\end{array}$ & 3,499 & 1.6 & 0.1 \\
$\begin{array}{l}\text { Dose } \\
\text { reported as } \\
\text { unknown }\end{array}$ & 1,421 & 4.4 & 2.6 \\
\hline
\end{tabular}

${ }^{\mathrm{a}}$ The lowest dose associated with an ANE ( $n=1$ in the up to $10 \mathrm{mg} / \mathrm{kg}$ group) was $9.6 \mathrm{mg} / \mathrm{kg}$

tions drawn from this data could assist in making a very similar clinical decision regarding HCF referral based only on a provided history of ingestion.

For cases when the dose was reported as unknown, these cases had what most centers would believe to be an unacceptable rate of ANE of $2.6 \%$, thus suggesting the need for referral for all these cases to a HCF. However, it appears that one can make an informed decision about HCF referral when a dose calculation in $\mathrm{mg} / \mathrm{kg}$ is possible. Over $75 \%$ of the total cases were already at or were sent to a HCF, but of these, 1,413 of the cases had a reported exposure amount of less than $10 \mathrm{mg} / \mathrm{kg}$, an amount associated with a $1.4 \%$ rate of moderate/major outcomes and a vanishingly small number of neurological events $(n=$ 1). Further inspection of the $10 \mathrm{mg} / \mathrm{kg}$ group revealed that except the one case with ANE (a dose of $9.6 \mathrm{mg} / \mathrm{kg}$ ), they were all $(n=34)$ moderate outcomes. The most common clinical effects reported with these cases were mild (tachycardia, agitated/irritable, drowsiness, ataxia, hallucinations, lethargy, and tremor), and should these develop, the patient could easily be referred to a HCF without any increase in morbidity from the ingestion. The author's conclusion for a clinically significant amount for $\mathrm{HCF}$ referral after a reported pediatric bupropion ingestion is a

Table 2 Dose estimations

\begin{tabular}{lccc}
\hline & Number of cases & Number of cases with mg/kg estimate & Average mg/kg \\
\hline No effect/mild outcome & $5,765(96.1 \%)$ & 4,406 & $13.8 \pm 18.8$ \\
Moderate/major outcome & $199(3.3 \%)$ & 137 & $38.8 \pm 44.0^{*}$ \\
Adverse neurological events (ANE) & $87(1.5 \%)$ & 50 & $73.6 \pm 54.3 * *$ \\
All cases & 5,964 & 4,543 & $14.5 \pm 20.5$ \\
\hline
\end{tabular}

$*_{p}<0.0001$ comparing no/mild effect

${ }^{* *} p<0.0001$ comparing no/mild effect and moderate/major effect 
$10 \mathrm{mg} / \mathrm{kg}$ amount. Further justification for this choice is that this is an easily remembered amount (less recall error by a specialist), it is less likely to result in mathematical error, only one in 2,465 cases had a seizure at this reported dose, and the clinical effects reported in this dose range were mostly mild. A recent study of 407 children after unintentional bupropion ingestion also suggested that a dose of $10 \mathrm{mg} / \mathrm{kg}$ or less could be safely managed at home [8]. Although there was some overlap in the two studied patient populations, this is similar to and supportive of our findings. This suggests that, depending on how much risk a poison center is willing to accept, a significant number children may be observed at home after a single substance bupropion exposure in lieu of HCF referral. The use of this dose as a triage guideline might result in $31 \%$ of cases that have been historically referred to be managed by home observation.

Once the patient is referred, observation must be for several hours to ensure that they will not develop any severe clinical effects, the most concern being seizures. Based on the average of the time until seizure, one would expect that observation of $8 \mathrm{~h}$ from ingestion time will be sufficient for over two-thirds of those patients that will develop seizures. But, the maximum time until seizures developed was $14 \mathrm{~h}$, a time span too long for most emergency department outpatient observation. Because the clinical effects timing is not recorded, it is impossible to say with certainty that these children were symptomatic before the seizure occurred, although a case review suggested that many seized before presenting or were symptomatic before the seizure. It is realized that there are two different extended release preparations of bupropion, and ideally, one would break the analysis into three groups to determine the outcomes and seizure time. But as discussed, this could not be reliably performed using this data set. It is known that the immediate release is associated with an increased rate of seizures [9]; this fact could be of assistance in making close clinical decisions based on this paper's $\mathrm{mg} / \mathrm{kg}$ information, which utilized a grouping of all preparation types.

The conclusions of this paper should be tempered with the retrospective nature of the study and the inherent limitations of poison center data. In the majority of patients, there was no independent clinical or laboratory verification that the bupropion ingestion had occurred. Of course, there is almost no opportunity to verify the ingestion of bupropion by laboratory analysis in a clinically relevant time span. Additionally, the inclusion criteria were limited to cases with follow-up; cases not followed up could have had a significantly adverse outcome, and it is impossible to know how many there were given that these were excluded during the initial data query. However, the large data set used in this study, covering more than 7 years and from a poison center system covering the entire US population, suggests that a reasonable decision can be made based on the data.

Few children develop toxicity from reported unintentional bupropion ingestions, with about $3.3 \%$ of pediatric reported ingestion exposures resulting in a moderate or major outcome, and only $1.5 \%$ of the cases reporting seizures or coma. It appears that referral to a HCF should be considered for all children where a reported dose could not be calculated (unknown) and children with calculated reported doses of greater than $10 \mathrm{mg} / \mathrm{kg}$ bupropion; a reported dose only associated with one ANE in 2,465 patients.

\section{References}

1. Micromedex ${ }^{\circledR}$ healthcare series, Thomson Reuters, Greenwood village, Colorado. Accessed February 2007

2. Instructions for the American Association of Poison Control Centers Toxic Exposure Surveillance System, Copyright AAPCC, Printed 2001

3. CDC (2009) http://www.cdc.gov/growthcharts. Published May 30, 2000, modified April 20, 2001

4. Stahl SM, Pradko M, Haight B, Modell JG, Rockett CB, LearnedCoughlin S (2004) Review of the neuropharmacology of bupropion. J Clin Psych 6(4):159-166

5. Balit C, Lynch CN, Isbister GK (2003) Bupropion poisoning: a case series. MJA 178(2):61-63

6. Belson MG, Kelly TR (2002) Bupropion exposures: clinical manifestations and medical outcome. J Emerg Med 23(3):223-230

7. Shepherd G, Velez LI, Keyes DC (2004) Intentional bupropion overdoses. J Emerg Med 27(2):147-151

8. Spiller HA, Bosse GM, Beuhler M, Gray T, Baker SD (2010) Unintentional ingestion of bupropion in children. J Emerg Med (in press)

9. Welbutrin XL (2007) Package insert. GlaxoSmithKline, Research Triangle Park 